\title{
Ultrafast IR spectroscopy of the short-lived transients formed by UV excitation of cytosine derivatives $\dagger$
}

\author{
Susan Quinn, ${ }^{* a}$ Gerard W. Doorley, ${ }^{a}$ Graeme W. Watson, ${ }^{a}$ Alexander J. Cowan, ${ }^{b}$ Michael W. George, ${ }^{b}$ \\ Anthony W. Parker, ${ }^{* c}$ Kate L. Ronayne, ${ }^{c}$ Michael Towrie ${ }^{c}$ and John M. Kelly ${ }^{* a}$
}

Received (in Cambridge, UK) 6th March 2007, Accepted 24th April 2007

First published as an Advance Article on the web 3rd May 2007

DOI: $10.1039 / b 703344 c$

A strong infrared band at $1574 \mathrm{~cm}^{-1}$ is observed following $267 \mathrm{~nm}$ excitation of $2^{\prime}$-deoxycytidine $(\tau=37 \pm 4 \mathrm{ps})$ or $2^{\prime}$-deoxycytidine 5'-monophosphate ( $\tau=33 \pm 4 \mathrm{ps}$ ); this band is provisionally attributed to an ${ }^{1} n_{N} \pi *$ state and is absent for cytosine.

The nature of the electronic excited states of DNA continues to be a focus of significant interest because of their role in UV-light induced modifications that could potentially be mutagenic. ${ }^{1}$ The nucleobases fluoresce extremely weakly and this has led to the conclusion that their excited states must be very short-lived. ${ }^{2}$ This is supported by femtosecond transient visible absorption ${ }^{3}$ and fluorescence upconversion ${ }^{4}$ measurements that reveal that the bases and mononucleotides possess sub-picosecond excited state lifetimes. However, the reasons for the short lifetimes are still a matter of debate, with theoretical studies emphasising the importance of ultrafast non-radiative processes. ${ }^{5}$ IR methods, including 2D-IR, are proving an increasingly valuable tool for the study of DNA systems. ${ }^{6,7}$ Time-resolved IR (TRIR) absorption spectroscopy $^{8}$ can provide structural as well as kinetic information on the ultrafast processes in DNA and our first paper in this area allowed the observation of the vibrationally excited ground states formed from nucleotides excited at $267 \mathrm{~nm}$. ${ }^{9}$ Subsequently the method has been used to identify the products of the photoionisation of GMP after excitation at $200 \mathrm{~nm}^{10}$ and very recently the formation of thymine dimers. ${ }^{11}$ In this paper we probe the transient species formed from $2^{\prime}$-deoxycytidine $5^{\prime}$-monophosphate (dCMP), 2'-deoxycytidine (dCyd) and cytosine (Cyt) using vibrational spectroscopy. The presence of a new short-lived species is shown for both dCyd and dCMP but not for the nucleobase Cyt.

Fig. 1 shows the transient IR difference spectra recorded between 2 ps and 1000 ps after $267 \mathrm{~nm}$ (150 fs) excitation of the mononucleotide dCyd in neutral (buffered $\mathrm{pH}$ 6.9) solution. (Identical spectra were also recorded at $\mathrm{pH} 8.5$ ). The spectrum shows areas corresponding to ground state depletion (bands at $1505,1524,1617$ and $1652 \mathrm{~cm}^{-1}$ as found in the FTIR) and ones

${ }^{a}$ School of Chemistry and Centre for Chemical Synthesis and Chemical Biology, Trinity College,Dublin 2,Ireland.E-mail: jmkelly@tcd.ie; quinnsu@tcd.ie; Fax:0035316712826; Tel: 0035318961947

${ }^{5}$ School of Chemistry, University of Nottingham, University Park,

Nottingham, UK

${ }^{c}$ Central Laser Facility, STFC Rutherford Appleton Laboratory,

Chilton, Didcot, Oxfordshire, UK OX11 0QX.

E-mail:A.W.Parker@rl.ac.uk

$\dagger$ Electronic supplementary information (ESI) available: Experimental

details and computational calculations. See DOI: 10.1039/b703344c where the transient absorbs more strongly. Similar behaviour is observed for dCMP (see ESI).

The transient decay (and the ground state recovery) is essentially complete over the 1000 ps time scale of the experiment. However, in contrast to the results for other nucleotides such as dAMP and dGMP, ${ }^{9}$ the kinetics are distinctly biphasic (see Fig. 2). At short times (2-8 ps - shown in red in Fig. 1) the transient absorption bands are observed to shift to higher wavenumber, behaviour which is consistent with relaxation of vibrationally excited ground states, as previously proposed. ${ }^{9}$ The time constant for this is determined to be $2.6 \pm 0.3$ ps for dCyd.<smiles>Nc1cc[nH]c(=O)n1</smiles><smiles>Cn1ccc(N)nc1=O</smiles>

cytosine (Cyt)

1-methylcytosine (1-MeCyt)<smiles>Nc1ccn([C@@H]2O[C@H](CO)[C@@H](O)[C@H]2O)c(=O)n1</smiles>

2'-deoxycytidine (dCyd)

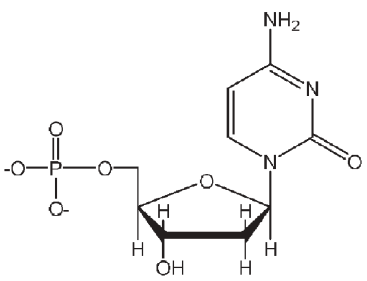

5'-dCMP

After this cooling process is complete a strong transient absorption at $1574 \mathrm{~cm}^{-1}$ persists. This band is the only one observable in the IR absorption spectrum in the region 1450 $1750 \mathrm{~cm}^{-1}$ (assuming that there is no accidental overlap of other bands with those of the ground state). This band decays on a longer time scale (average lifetime of $37 \pm 4$ ps) with the consequent reformation of the ground state of dCyd. Analogous behaviour is recorded for dCMP, with biphasic kinetics of $2.6 \pm$ 0.3 ps and $33 \pm 4$ ps (see ESI). However, when the measurements were repeated for the nucleobase Cyt (Fig. 1b) the transient species was found to undergo rapid single exponential decay with a lifetime of $4.4 \pm 0.4 \mathrm{ps}$, with no significant (i.e. $<5 \%$ ) longer-lived component being detectable.

We have considered various options for the relatively long-lived species of dCyd and dCMP - namely that it could be an excited state, a tautomer or another photoproduct.

It is known that the luminescence from dCMP is very short-lived $(<1 \mathrm{ps}),{ }^{4 b}$ so that the transient we observe cannot be a ${ }^{1} \pi-\pi^{*}$ singlet state. Furthermore, the triplet state is known 


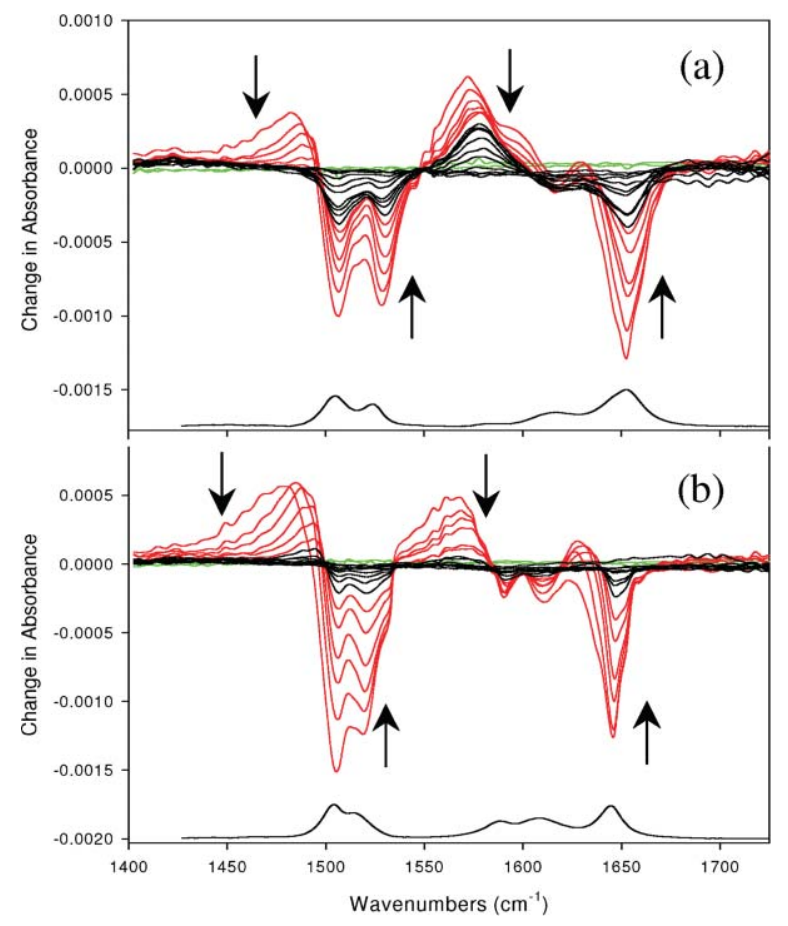

Fig. 1 ps-TRIR of (a) $20 \mathrm{mM}$ dCyd and (b) $20 \mathrm{mM}$ Cyt both in $50 \mathrm{mM}$ phosphate buffer $\mathrm{pH} 7$ with FTIRs below. Delays at $-50,-25$ (green), 2, $3,4,5,6.5,8$ (red), 10, 12.5, 15, 20, 35, 50, 100, 150, 200, 500 and 1000 ps (black).

to be relatively long-lived and at $10 \mathrm{mM}$ has a lifetime of $c a$. 250 ns. $^{12}$

The possibility that this species might be due to a protonated or deprotonated excited state has also been examined. However, the ps-TRIR transient behaviour we observe is the same at $\mathrm{pH} 6.9$ and $\mathrm{pH} 8.5$, as also are the reported transient visible data, ${ }^{13}$ so deprotonation is unlikely to be important. Both the ground state and transient behaviour for $\mathrm{dCMP}$ in acid are quite different from that recorded in neutral solution (see ESI). Here the transient absorption band shifts to higher wavenumber as the signal decays and shows a single exponential rapid recovery of the ground state (lifetime $=4.0 \pm 0.4 \mathrm{ps}$ ) - behaviour consistent with vibrational relaxation. This indicates that protonation suppresses the

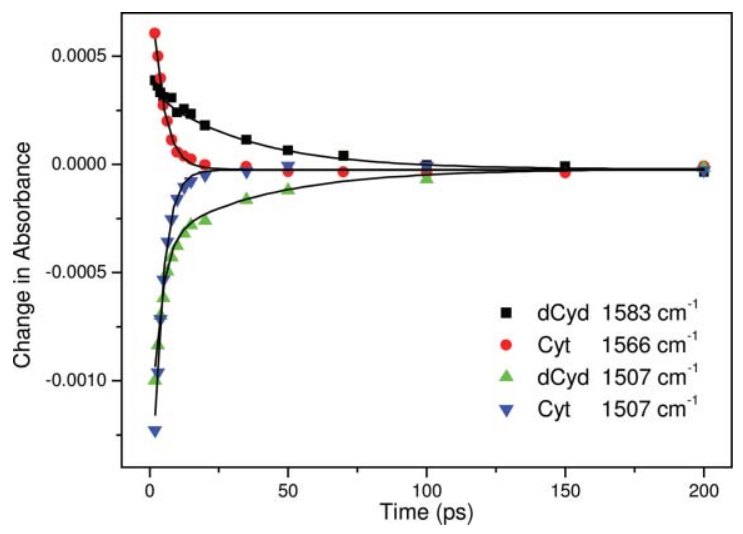

Fig. 2 Kinetic analysis of $20 \mathrm{mM} \mathrm{dCyd}$ at 1583 and $1507 \mathrm{~cm}^{-1}$ and $20 \mathrm{mM} \mathrm{Cyt}$ at 1566 and $1507 \mathrm{~cm}^{-1}$ in $50 \mathrm{mM}$ phosphate buffer $\mathrm{pH} 7$. formation of the $1574 \mathrm{~cm}^{-1}$ transient and provides an efficient route for radiationless deactivation.

These data led us initially to consider whether the $1574 \mathrm{~cm}^{-1}$ transient was a short-lived photoproduct. At the concentration used dimer formation is not expected to be important and these and other photoproducts such as 6-hydroxy-5,6-dihydrocytosine, the so-called cytosine photohydrate, ${ }^{14}$ are expected to be stable in the ps range. We considered the possibility of this species being a tautomer as there have been a number of theoretical studies of the tautomers of cytosine derivatives. ${ }^{15-20}$ For dCyd and dCMP the most stable tautomers are $\mathbf{I}$ or $\mathbf{H}$ and a recent theoretical study ${ }^{19}$ indicates that the imino-carbonyl isomer $\mathbf{I} \cdot \mathbf{H}_{\mathbf{2}} \mathbf{O}$ can rapidly revert to its canonical form $\mathbf{C} \cdot \mathbf{H}_{\mathbf{2}} \mathbf{O}$.

To identify whether the transient species could be one of these tautomers we performed optimisations and vibrational frequency calculation using both B3LYP and MP2 with a 6-311+G(2d,p) basis set in the Gaussian 03 package. ${ }^{21,22}$ For these preliminary studies we undertook calculations on 1-methylcytosine (and its hydrate $\mathbf{C} \cdot \mathbf{D}_{2} \mathbf{O}$ ) and determined the individual and difference spectra between the 1-methylcytosine and its tautomers (these are shown in the ESI). The calculations indicate that species I (or its hydrate $\mathbf{I} \cdot \mathbf{D}_{\mathbf{2}} \mathbf{O}$ ) should have a strong IR absorption band at higher frequency than $\mathrm{dCyd}$ in contrast with what is found experimentally. The absence of this band in the experiment therefore speaks against this tautomeric form. Calculations on species $\mathbf{H}$ give rise to spectra which do not have this high frequency peak but agreement with the experimental spectra is still not satisfactory. However, given the limitations of the calculations, e.g. explicit consideration of only a single water molecule bound as shown, we are unable to completely rule out the formation of the imino-enol tautomer $\mathbf{H}$.

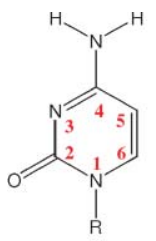

(C)<smiles>[2H]/N=c1\ccn(P)c(=O)[nH]1</smiles>

(I)<smiles>N=c1ccn(P)c(O)n1</smiles>

(H)<smiles></smiles>

$\left(\mathrm{I} . \mathrm{D}_{2} \mathrm{O}\right)$

$$
\mathrm{R}=\mathrm{H}, \mathrm{Cyt} ; \mathrm{CH}_{3}, 1-\mathrm{MeCyt}
$$

Having shown that the $1574 \mathrm{~cm}^{-1}$ transient is unlikely to be a transient photoproduct we next considered the possibility of the new species being a 'dark' excited state of dCyd, even though these had not been observed by detailed visible transient absorption studies. ${ }^{3,12}$ However, during the preparation of this manuscript the existence of such an intermediate dark state of dCMP was suggested from a kinetic analysis of the transient bleaching behaviour at $340 \mathrm{~nm} .{ }^{23}$ The authors tentatively assigned the species to a ${ }^{1} n \pi^{*}$ state. In the case of dCMP the lifetime of this component was found to be $34 \pm 3$ ps in excellent agreement with the results observed in our ps-TRIR experiments.

The observation of a single strong IR band in the transient spectra between 1500 and $1700 \mathrm{~cm}^{-1}$ is quite striking given the presence of multiple bands in the ground state in the same spectral window. In the ground state these bands correspond to vibrations of the carbonyl bond, ring double bond stretches and $\mathrm{NH}_{2}$ bending vibration. ${ }^{18,24}$ The simplicity of this transient spectrum may be due to a variety of structural and electronic changes. Such changes may result from the destruction of the $\pi$-conjugated 


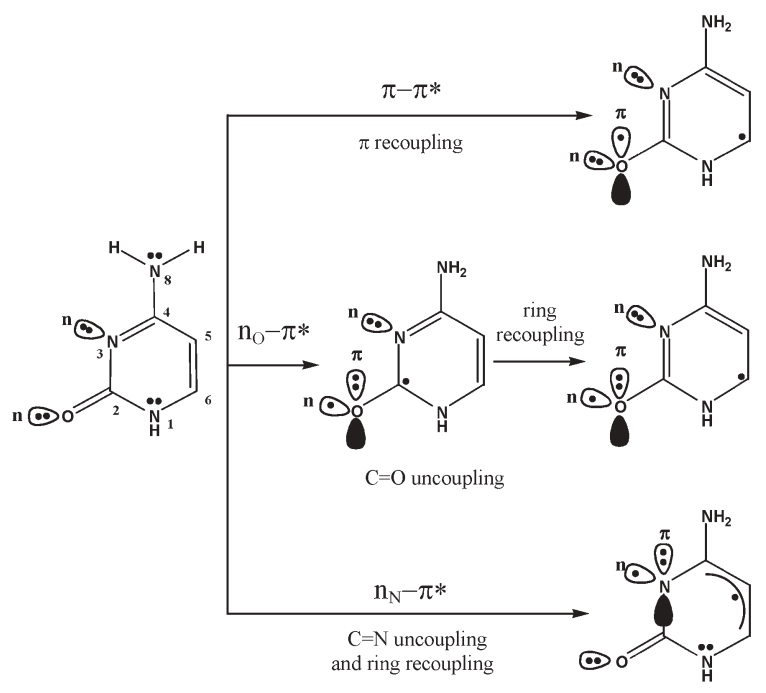

Scheme 1 Valence bond representation of singlet excited states of Cyt (after Ismail et al.). ${ }^{5 a}$

bonding network in the dCyd atomic frame causing loss of IR activity in this spectral region. While at present a definitive identification of the $1574 \mathrm{~cm}^{-1} \mathrm{dCyd}$ transient band is not possible, we provisionally assign it to the $\mathrm{C} 2=\mathrm{O}$ mode. Confirmation of this will require isotopic $\left({ }^{18} \mathrm{O}\right)$ substitution.

Two possible ${ }^{1} n \pi^{*}$ excited states (the ${ }^{1} n_{\mathrm{O}} \pi^{*}$ and ${ }^{1} \mathrm{n}_{\mathrm{N}} \pi^{*}$ ) should be considered for the cytosine derivatives, the energetics of which have been the subject of theoretical studies. ${ }^{25}$ However the presence of a strong band at $1574 \mathrm{~cm}^{-1}$ in the transient IR spectra may be a distinguishing feature, as valence bond treatment $^{5 a}$ of the two states (Scheme 1) predicts that there is substantial double bond character in the ${ }^{1} \mathrm{n}_{\mathrm{N}} \pi^{*}$ but not in the ${ }^{1} n_{\mathrm{O}} \pi^{*}$. In the case of ${ }^{1} n_{\mathrm{O}} \pi^{*}$ the valence bond treatment indicates substantial single bond character for the carbonyl bond and therefore its vibration is not expected to be close to $1574 \mathrm{~cm}^{-1}$. This strongly suggests that we are dealing with a ${ }^{1} n_{N} \pi^{*}$. Computational analysis to assist our assignment of the $1574 \mathrm{~cm}^{-1}$ requires the calculation of the second derivative matrix at an optimised excited-state geometry and requires substantial computational resources. We hope to report results from such calculations in a subsequent publication.

In summary we propose that the deactivation of dCMP and $\mathrm{dCyd}$ can proceed by at least two routes. The first of these involves the rapid formation of the vibrationally excited ground state of dCMP, which then relaxes with a lifetime of 2.6 ps to the 'cooled' ground state. The second process, which competes with this, proceeds via a species possessing a strong vibrational band at $1574 \mathrm{~cm}^{-1}$. If this species is a tautomer our calculations suggest that it is very unlikely to be the imino-carbonyl isomer $\mathbf{I}$. Although we cannot at this stage exclude the possibility that this species is the imino-enol isomer $\mathbf{H}$, we consider it more likely to be the ${ }^{1} n_{N} \pi^{*}$ state. The fact that no analogous species is formed upon excitation of the nucleobase, cytosine, demonstrates that substitution at the 1-position by 2-deoxyribose has a major effect on the photophysical properties and probably on the photochemical reactivity.

We thank EU FP 6 co-ordinated through LaserLabs Europe (CLF001281) for granting access to the Central Laser Facility, STFC Rutherford Appleton Laboratory. GWD thanks the Science Foundation Ireland RFP for financial support.

\section{Notes and references}

1 J. Cadet, S. Courdavault, J. L. Ravanat and T. Douki, Pure Appl. Chem., 2005, 77, 947.

2 M. Daniels and W. Hauswirth, Science, 1971, 171, 675.

3 (a) C. E. Crespo-Hernandez, B. Cohen, P. M. Hare and B. Kohler, Chem. Rev., 2004, 104, 1977; (b) J. M. L. Pecourt, J. Peon and B. Kohler, J. Am. Chem. Soc., 2001, 123, 10370.

4 (a) A. Sharonov, T. Gustavsson, V. Carr, E. Renault and D. Markovitsi, Chem. Phys. Lett., 2003, 380, 173; (b) D. Onidas, D. Markovitsi, S. Marguet, A. Sharonov and T. Gustavsson, J. Phys. Chem. B, 2002, 106, 11367.

5 (a) N. Ismail, L. Blancafort, M. Olivucci, B. Kohler and M. A. Robb, J. Am. Chem. Soc., 2002, 124, 6818; (b) L. Blancafort, B. Cohen, P. M. Hare, B. Kohler and M. A. Robb, J. Phys. Chem. A, 2005, 109, 4431.

6 E. Taillandier and J. Liquier, Vibrational Spectroscopy of the Nucleic Acids, in the Handbook of Vibrational Spectroscopy, ed. J. M. Chalmers and P. R. Griffiths, Wiley, Chichester, 2002.

7 A. T. Krummel and M. T. Zanni, J. Phys. Chem. B, 2006, 110, 13991.

8 M. Towrie, D. C. Grills, J. Dyer, J. A. Weinstein, P. Matousek, R. Barton, P. D. Bailey, N. Subramaniam, W. M. Kwok, C. S. Ma, D. Phillips, A. W. Parker and M. W. George, Appl. Spectrosc., 2003, 57, 367.

9 M. K. Kuimova, J. Dyer, M. W. George, D. C. Grills, J. M. Kelly, P. Matousek, A. W. Parker, X. Z. Sun, M. Towrie and A. M. Whelan, Chem. Commun., 2005, 1182.

10 M. K. Kuimova, A. J. Cowan, P. Matousek, A. W. Parker, X. Z. Sun, M. Towrie and M. W. George, Proc. Natl. Acad. Sci. U. S. A., 2006, 103, 2150.

11 W. J. Schreier, T. E. Schrader, F. O. Koller, P. Gilch, C. E. CrespoHernandez, V. N. Swaminathan, T. Carell, W. Zinth and B. Kohler, Science, 2007, 315, 625.

12 (a) I. G. Gut, P. D. Wood and R. W. Redmond, J. Am. Chem. Soc., 1996, 118, 2366; (b) Z. Zuo, S. Yao, J. Luo, W. Wang, J. Zhang and N. Lin, J. Photochem. Photobiol., B, 1992, 15, 215.

13 R. J. Malone, A. M. Miller and B. Kohler, Photochem. Photobiol., 2003, 77, 158.

14 (a) F. T. Liu and N. C. Yang, Biochemistry, 1978, 17, 4877; (b) G. DeBoer and H. E. Johns, Biochim. Biophys. Acta, 1970, 204, 18; (c) G. DeBoer, O. Klinghoffer and H. E. Johns, Biochim. Biophys. Acta, 1970, 213, 253.

15 G. Fogarasi, J. Phys. Chem. A, 2002, 106, 1381.

16 M. Szczesniak, K. Szczepaniak, J. S. Kwiatkowski, K. KuBalat and W. B. Person, J. Am. Chem. Soc., 1988, 110, 8319.

17 I. R. Gould, M. A. Vincent, I. A. Hiller, L. Lapinski and M. J. Nowak, Spectrochim. Acta, Part A, 1992, 48A, 811.

18 N. Leulliot, M. Ghomi, H. Jobic, O. Bouloussa, V. Baumruk and C. Coulombeau, J. Phys. Chem. B, 1999, 103, 10934.

19 Y. Podolyan, L. Gorb and J. Leszczynski, Int. J. Mol. Sci., 2003, 4, 410.

20 G. N. Ten and V. I. Baranov, J. Appl. Spectrosc., 2005, 72, 155.

21 M. J. Frisch, et al., Gaussian 03 Revision C.02; Gaussian, Inc.: Pittsburgh, PA, 2003.

22 M. P. Andersson and P. Uvdal, J. Phys. Chem. A, 2005, 109, 2937.

23 P. M. Hare, C. E. Crespo-Hernandez and B. Kohler, Proc. Natl. Acad. Sci. U. S. A., 2007, 104, 435.

24 C. Lee, K.-H. Park and M. Cho, J. Chem. Phys., 2006, 125, 114508.

25 L. Blancafort, Photochem. Photobiol., DOI: 10.1562/2006-05-29-RA-903 and refs. therein. 